\title{
Coexistence of Histologically Confirmed Hashimoto's Thyroiditis with Different Stages of Papillary Thyroid Carcinoma in a Consecutive Chinese Cohort
}

\author{
Xiaoyun Liu, ${ }^{1}$ Lijun Zhu, ${ }^{2}$ Dai Cui, ${ }^{1}$ Zhixiao Wang, ${ }^{1}$ Huanhuan Chen, ${ }^{1}$ \\ Yu Duan, ${ }^{1}$ Meiping Shen, ${ }^{3}$ Yunsong Wu, ${ }^{4}$ Rong Rong, ${ }^{4}$ Zhihong Zhang, ${ }^{4}$ Xiaodong Wang, \\ Jiawei Chen, ${ }^{1}$ Erik K. Alexander, ${ }^{5}$ and Tao Yang ${ }^{1}$ \\ ${ }^{1}$ Department of Endocrinology, First Affiliated Hospital of Nanjing Medical University, Nanjing, Jiangsu 210029, China \\ ${ }^{2}$ Department of Children's Health Care, Nanjing Maternity and Child Health Care Hospital Affiliated to Nanjing Medical University, \\ Nanjing, Jiangsu 210029, China \\ ${ }^{3}$ Department of Surgery, First Affiliated Hospital of Nanjing Medical University, Nanjing, Jiangsu 210029, China \\ ${ }^{4}$ Department of Pathology, First Affiliated Hospital of Nanjing Medical University, Nanjing, Jiangsu 210029, China \\ ${ }^{5}$ Thyroid Unit, Division of Endocrinology, Metabolism and Diabetes, Department of Medicine, \\ Brigham \& Women's Hospital and Harvard Medical School, Boston, MA 02115, USA
}

Correspondence should be addressed to Tao Yang; yangt@njmu.edu.cn

Received 31 May 2014; Revised 25 October 2014; Accepted 4 November 2014; Published 18 November 2014

Academic Editor: Jack R. Wall

Copyright (C) 2014 Xiaoyun Liu et al. This is an open access article distributed under the Creative Commons Attribution License, which permits unrestricted use, distribution, and reproduction in any medium, provided the original work is properly cited.

\begin{abstract}
Purpose. To determine the relationship between Hashimoto's thyroiditis (HT) and all stages of papillary thyroid carcinoma (PTC) with or without local lymph node metastasis (LNM). Methods. We conducted a retrospective study of thyroidectomies from 2008-2013 in First Affiliated Hospital of Nanjing Medical University. We categorized patients according to the presence of histopathologically proven HT. The prevalence of mPTC (maximum diameter $\leq 10 \mathrm{~mm}$ ) and crPTC (clinical relevant PTC) and local LNM rates were compared. Results. We evaluated 6,432 consecutive thyroidectomies. In total, 1,328 specimens were confirmed as HT. The prevalence of PTC in this HT cohort was 43.8\%, significantly higher than non-HT group. After adjustment of gender and age, the prevalence of PTC was still higher in HT group. HT was a risk factor for PTC in multivariate analysis with odds ratio 2.725 (95\% CI, 2.390-3.109) $(P<0.001)$. However, no correlation was found between HT and LNM of PTC. Conclusion. HT was associated with an increased prevalence of all stages of PTC, independent of tumor size, gender, and age. In contrast, locally advanced disease defined by LNM was unrelated to HT. These data suggest an association of HT with low risk PTC and a potential protective immunologic effect from further disease progression.
\end{abstract}

\section{Introduction}

Thyroid cancer is the most common endocrine malignancy worldwide, with a significant increase in global incidence [1$4]$ over the past decade. This is true both in the United States $[2,5]$ and in eastern Asia including China [6-9]. About 70\% to $80 \%$ of thyroid cancers are papillary thyroid carcinoma (PTC) $[9,10]$. Despite surgical advances and better long-term results, however, the underlying pathogenesis of PTC remains unclear.

Hashimoto's thyroiditis (HT) has been postulated to have a causative relationship to PTC, being suggested as a possible risk factor for developing PTC. This link was first proposed in 1955 with several subsequent retrospective studies showing similar results [11-15]. The exact mechanism remains uncertain. Some studies suggest that stimulating TSH concentrations (often mildly elevated) may be one responsible factor for this relationship [16]. In support, treatment with $\mathrm{L}-\mathrm{T}_{4}$ may reduce TSH levels and decreases the prevalence of clinically detectable PTC [12]. However, other studies are contradictory finding TSH concentration similar among patients regardless of PTC or benign disease [17].

It is well known that PTC represents a spectrum of disease inclusive of heterogeneity and severity in histologic patterns 
and/or tumor stages. Occult or microscopic PTC (mPTC) is frequently incidentally detected in histologic samples even when benign nodules are assumed. It is uncertain whether incidental micro-PTC (mPTC) simply represents an early stage of overt (or clinically relevant) PTC or is actually a different subtype of PTC which seldom progresses. It is therefore reasonable to postulate that the analysis of PTC as a unified single group may in part be responsible for the conflicting published data. Notably, most previous studies only evaluated clinically relevant PTC (crPTC, maximum diameter $>1 \mathrm{~cm}$ in size). We hypothesize that $\mathrm{mPTC}$ might be the early stage of crPTC with similar relationships existing between MPTC and HT when compared to crPTC and HT. However, some evidence suggests that the presence of HT may be associated with less risk of PTC lymph node metastasis (LNM) and an overall better prognosis [11, 18]. To elucidate the uncertainty as to the influence of HT upon local tumor growth (even from a very early stage), we conducted a retrospective study trying to clarify the relationship between HT and different stages of PTC.

\section{Methods}

We collected data from the Register and Reporting system in Department of Pathology, the First Affiliated Hospital of Nanjing Medical University. Particularly, we reviewed all consecutive thyroid histopathological reports from January 2008 to December 2013. Patient demographic data was obtained by chart review at a central location by Xiaoyun Liu, Lijun Zhu, Dai Cui, Zhixiao Wang, Huanhuan Chen, Yu Duan, Xiaodong Wang, and Jiawei Chen.

Generally, thyroidectomies were performed for the following reasons: (a) worrisome findings from ultrasonography and/or abnormal lymph node enlargement, (b) malignancy suspected from previous thyroid FNA, or inconclusive FNA results, (c) patients with multiple or bilateral nodules or symptoms of neck or throat compression, or enlargement during follow-up, and (d) concerning clinical or physical examination findings warranting consideration for removal. A small minority of patients chose thyroidectomy at their own wish, primarily for cosmetic reasons.

Malignancy, if present, was reported as one of the following subtypes: crPTC, with the maximum diameter larger than $10 \mathrm{~mm}$; mPTC, with the maximum diameter less than or equal to $10 \mathrm{~mm}[19,20]$; follicular thyroid carcinoma (FTC); medullary thyroid carcinoma (MTC); and anaplastic thyroid carcinoma (ATC). For each case, we collected data confirming the maximum diameter of the tumor, tumor multifocality, and other pathologic features. The impact of Hashimoto's thyroiditis upon mPTC and crPTC rates was the primary focus of this investigation. Papillary structures sometimes seen in nodular hyperplasia were not recorded as PTC. All pathological findings were confirmed by three experienced endocrine pathologists (Yunsong $\mathrm{Wu}$, Rong Rong, and Zhihong Zhang).

Lymph node dissection was performed at the discretion of the surgeon based upon clinical and other factors according to the guideline from the American Thyroid Association
(ATA) [21]. Most often, a neck ultrasound or computed tomography (CT) scan was performed prior to surgery allowing assessment of neck adenopathy. Frozen sections were routinely used to guide the extent of the surgical procedures. If the nodule was found to be malignant by frozen section and no abnormal lymph nodes were identified on preoperative imaging (or during the surgery), an ipsilateral central lymph node dissection (CLND) from level VI was generally performed. Only when the tumor size was within $10 \mathrm{~mm}$ and no abnormal lymph node was found during the operation, CLND was not routinely performed. If abnormal lymph nodes were identified during preoperative ultrasound or intraoperatively, and the snap-frozen sample showed malignant lesion, a modified lateral lymph node dissection (LLND) was performed. All the samples were formalin fixed paraffin embedded (FFPE) for final histopathology confirmation.

The histologic diagnosis of HT was made according to accepted standards [18]. HT was defined as the presence of diffuse lymphocytic infiltration with the formation of lymphoid follicles and the presence of reactive germinal centers; the infiltrate had to occur in a normal region of the thyroid gland, distinct from the site of the thyroid carcinoma. Peritumoural inflammatory response and small areas of lymphocytic infiltration were not designated as HT. The diagnosis of HT was solely made upon the histological findings. The antithyroglobulin and antithyroid peroxidase antibody levels were not routinely measured for the diagnosis of HT.

We recruited 6,432 cases with valid pathology reports including all thyroidectomies performed over the six-year period. We next categorized each according to the histologic diagnosis of $\mathrm{HT}$ and separately for the identification of $\mathrm{mPTC}$ and crPTC (or other cancer types).

Quantitative data are shown as mean \pm SD, compared using independent samples $t$-test, whereas numbers and percentage are provided for qualitative data. Percentages were compared using the $\chi^{2}$ test. The odds ratio (OR) and the $95 \%$ confidence interval (CI) for relationships between each variable and PTC or positive LNM (yes or no) were calculated using binary logistic regression. All tests were 2 -sided, and a $P$ value $<0.05$ was considered statistically significant. Statistical analyses were performed with SPSS software, version 13.0 for Windows (SPSS Inc., Chicago, IL, USA).

This study was reviewed and deemed exempt from written informed consent by the Institutional Review Board (IRB) of the First Affiliated Hospital of Nanjing Medical University. It was approved by the IRB for analysis.

\section{Results}

3.1. Basic Characteristics and Thyroid Carcinoma Distribution of Non-HT and HT Groups. Over a 6-year period, we evaluated 6,432 consecutive patients who underwent thyroidectomy. Demographic and pathologic details for all subjects are shown in Table 1. Among this cohort, 20.6\% $(1,328 / 6,432)$ of our thyroidectomy population was histologically confirmed to have HT. Not surprisingly, those with HT were disproportionately female, consistent with other autoimmune illnesses. The mean age of the HT cohort was 
TABLE 1: Baseline characteristics and thyroid carcinoma distribution between non-HT and HT groups.

\begin{tabular}{lccr}
\hline & Non-HT $(n=5104)$ & HT $(n=1328)$ & $\chi^{2}$ \\
\hline Age at diagnosis & $48.72 \pm 13.74$ & $46.21 \pm 13.55$ & $<0.01$ \\
$<45$ y $(n=2565)$ & $1972(38.6 \%)$ & $593(44.7 \%)$ & 15.914 \\
$\quad \geqslant 45$ y $(n=3867)$ & $3132(61.4 \%)$ & $735(55.3 \%)$ & \\
Gender & & & \\
Male $(n=1373)$ & $1274(25.0 \%)$ & $99(7.5 \%)$ & 192.350 \\
Female $(n=5059)$ & $3830(75.0 \%)$ & $1229(92.5 \%)$ & 246.051 \\
PTC $(n=1722)$ & $1141(22.4 \%)$ & $581(43.8 \%)$ & 106.826 \\
mPTC $(n=823)$ & $541(10.6 \%)$ & $282(21.2 \%)$ & 101.467 \\
crPTC $(n=899)$ & $600(11.8 \%)$ & $299(22.5 \%)$ & 1.783 \\
FTC $(n=115)$ & $97(1.9 \%)$ & $18(1.4 \%)$ & 0.173 \\
MTC $(n=34)$ & $26(0.5 \%)$ & $8(0.6 \%)$ & $<0.01$ \\
ATC $(n=4)$ & $3(0.1 \%)$ & $1(0.1 \%)$ & $<0.01$ \\
MC $(n=841)$ & $556(43.9 \%)$ & $285(46.6 \%)$ & 1.04 \\
Other cancer $(n=101)$ & $86(1.7 \%)$ & $15(1.1 \%)$ & 0.18 \\
All cancer $(n=1977)$ & $1354(26.5 \%)$ & $623(46.9 \%)$ & 0.68 \\
Total $(n=6,432)$ & 5104 & 1328 & 0.83 \\
\hline
\end{tabular}

Data are expressed as number (percentage) or mean \pm SD unless otherwise specified.

TABLE 2: Univariate and multivariate analysis for PTC.

\begin{tabular}{|c|c|c|c|c|}
\hline \multirow{2}{*}{ Independent variable } & \multicolumn{2}{|c|}{ Univariate } & \multicolumn{2}{|c|}{ Multivariate } \\
\hline & OR (95\% CI) & $P$ value & OR & $P$ value \\
\hline Age $(\geqslant 45$ versus $<45)$ & $0.469(0.420-0.525)$ & 0.001 & $0.474(0.423-0.532)$ & 0.001 \\
\hline Gender (male versus female) & $0.906(0.790-1.039)$ & 0.157 & $1.153(0.999-1.330)$ & 0.051 \\
\hline Hashimoto thyroiditis (HT versus non-HT) & $2.701(2.380-3.067)$ & 0.001 & $2.725(2.390-3.109)$ & 0.001 \\
\hline
\end{tabular}

$46.2 \pm 13.6$ years, significantly younger than the non-HT group.

A total of 1,977 thyroidectomy specimens $(30.7 \%$ of 6,432$)$ were shown to harbor malignancy-mostly PTC (87.1\% of all malignancy). However, 823 cases ( $41.6 \%$ of all malignancy) were best classified as mPTC and 899 specimens ( $45.5 \%$ of all malignancy) demonstrated a clinically relevant $(>1 \mathrm{~cm})$ papillary thyroid carcinoma. A low but detectable rate of FTC, MTC, and ATC was also identified, but these rates were not different in the HT cohort versus others (Table 1).

We next determined the prevalence of thyroid malignancy in thyroidectomy specimens with or without histologic evidence of HT. In the HT group, $46.9 \%$ specimens were found to also contain a thyroid malignancy of any pathological type, compared to $26.5 \%$ of those without HT $(P<0.01$, Table 1). Interestingly, however, the difference in malignancy rate was solely attributable to differences in the proportion of PTC in the two cohorts. Of the 823 patients with mPTC, $541(10.6 \%)$ patients were in the non-HT group while 282 (21.2\%) patients were in HT group (Table 1). This finding was consistent for crPTC as well. No difference was detected in the rate of FTC, MTC, or other malignancies (Table 1).

3.2. Prevalence of PTC (including $m P T C$ and crPTC) in Various Subgroups. Due to the gender and age discrepancy in non-HT and HT group, we divided the total population into four subcategories according to the sex and age differences. It was shown consistently that the prevalence of PTC was higher in all Hashimoto's cohorts in comparison with non-HT groups (Figure 1(a) and supplementary Table 1 in Supplementary Material available online at http://dx.doi.org/10.1155/ 2014/769294). Nearly all subgroups analysis showed similar statistical findings for the prevalence of mPTC with the exception of male under the age of 45 years (Figure 1(b) and supplementary Table $1, P=0.05$ ) and for the prevalence of crPTC with one exception in male $\geqslant 45$ years (Figure $1(c)$ and supplementary Table 1).

3.3. Risk Factor for PTCs. We used univariate and multivariate analysis to determine whether the presence of HT would be an independent risk factor for PTC. As it was shown in Table 2, HT was a significant independent risk factor for PTC with odds ratio 2.725 (95\% CI, 2.390-3.109). The age $\geqslant 45$ years was a protective risk factor for PTC with odds ratio 0.474 (95\% CI, 0.423-0.532).

3.4. Local Lymph Node Metastasis (LNM) between Groups. We next investigated the association of histologic HT upon local metastatic disease as defined by lymph node metastasis (LNM). Inclusive of all thyroid malignancy identified over 6 years, the presence of HT showed a neutral effect upon local LNM when thyroid cancer was present $(P>0.05$, Figure 2 and supplementary Table 2). 


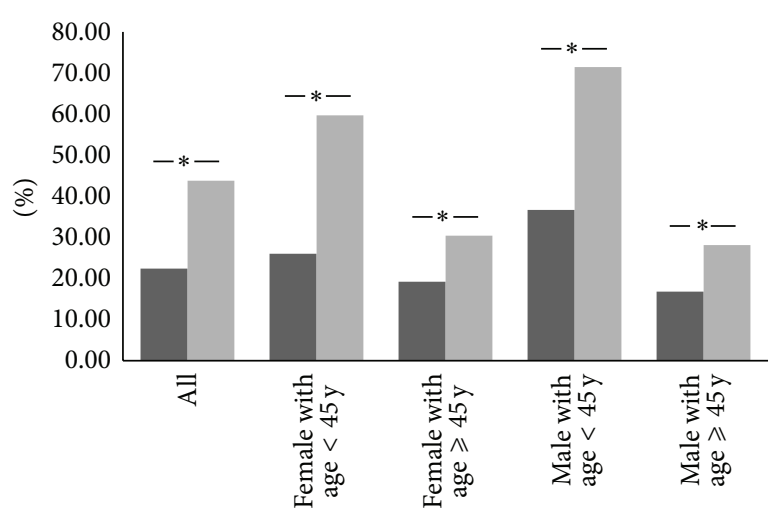

(a)

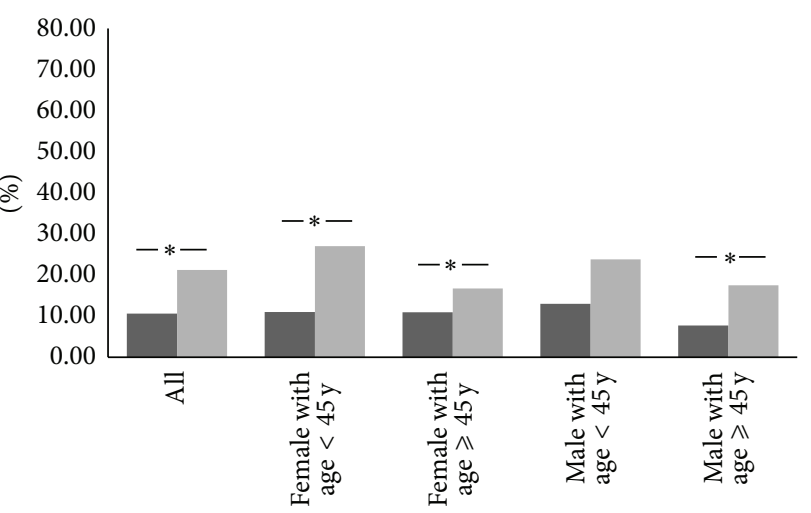

(b)

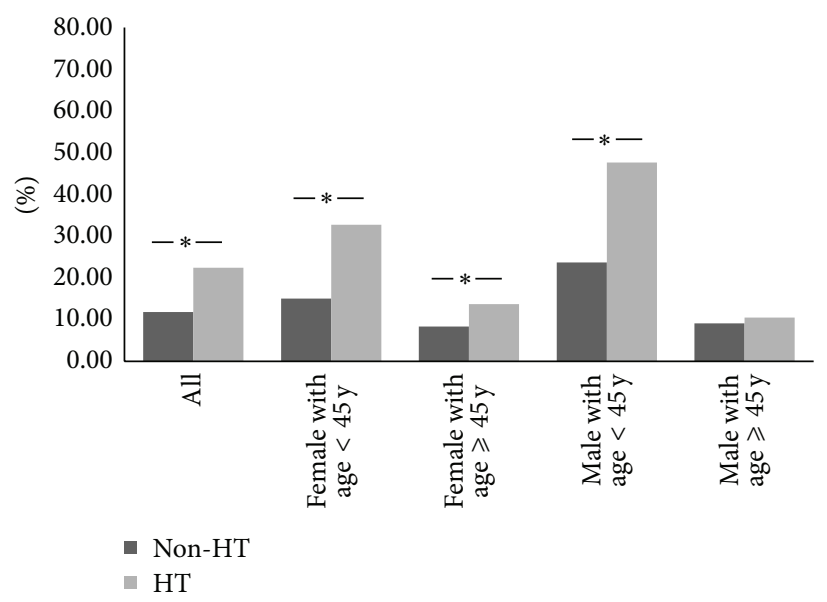

(c)

Figure 1: The prevalence of PTC (a), mPTC (b), and crPTC (c) in different age and gender groups. ${ }^{*} P<0.05$.

3.5. Risk Factors for LNM in PTCs. We then determined the risk factors of LNM in PTCs. As shown in Table 3, we found that male gender, age $<45$ years, foci of tumor, and maximum diameter were risk factors for LNM in PTCs, while HT was neither a protective nor risk factor for LNM in PTCs with OR 0.978, 95\% CI (0.772-1.239) (Table 3).

3.6. Basic Characteristics of All PTCs between Non-HT and HT Groups. All cohorts evaluated with PTC demonstrated an association with younger age and female predominance in the Hashimoto's cohort compared with non-HT groups. This was independent of tumor size (Table 4). No difference was noted in the number of positive lymph nodes or the number of specific tumor foci between HT and non-HT groups, respectively. Interestingly, in all patients with Hashimoto's disease, the size of all identified clinically relevant (crPTC) was generally smaller compared to those without Hashimoto's disease. In contrast, mPTCs were more often bilateral (though not more often multifocal) in the HT cohort compared to nonHT cohort.

\section{Discussion}

The relationship between Hashimoto's thyroiditis and thyroid malignancy has long been controversial. Differences in study

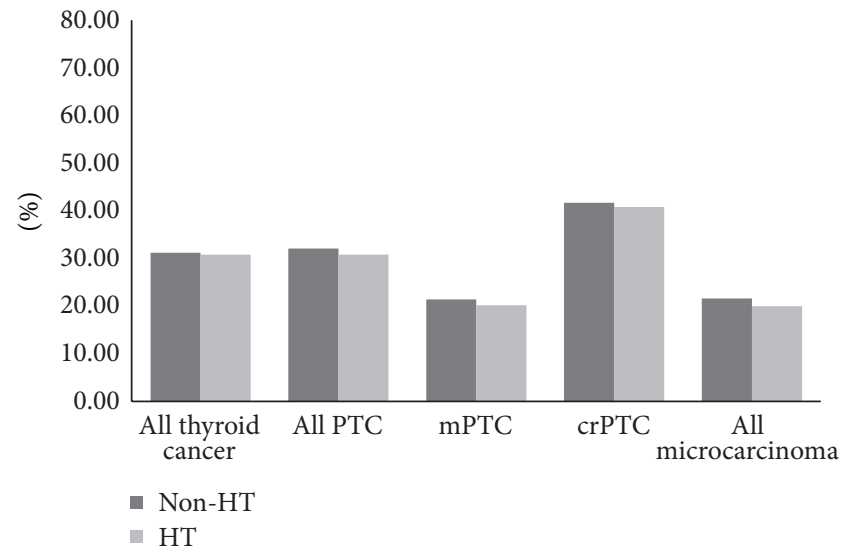

Figure 2: Local lymph node metastasis in different groups. No significance was found between HT and non-HT groups.

design, selection bias, and standardized approaches have led to a mixed literature in this regard. Arguably, to best investigate this important association, a large cohort of consecutive patients must be studied and all diagnoses-such as Hashimoto's disease and thyroid carcinoma-defined by expert histopathologic analysis. 
TABLE 3: Univariate and multivariate analysis for LNM in PTCs.

\begin{tabular}{lccc}
\hline Independent variable & Univariate & & $\begin{array}{c}\text { Multivariate } \\
P \text { value }\end{array}$ \\
\hline Age $(\geqslant 45$ versus $<45)$ & OR $(95 \%$ CI $)$ & $P$ value & 0.001 \\
Gender (Male versus Female) & $0.439(0.355-0.542)$ & 0.001 & $0.455(0.363-0.569)$ \\
Hashimoto thyroiditis (HT versus non-HT) & $1.723(1.351-2.197)$ & 0.001 & $1.544(1.181-2.017)$ \\
Foci of tumor & $0.943(0.760-1.170)$ & 0.593 & $0.978(0.772-1.239)$ \\
Maximum diameter & $1.547(1.367-1.750)$ & 0.001 & $1.471(1.293-1.673)$ \\
\hline
\end{tabular}

TABLE 4: Baseline data of PTC (mPTC and crPTC) between non-HT and HT group.

\begin{tabular}{|c|c|c|c|c|}
\hline & Non-HT & $\mathrm{HT}$ & Total & $P$ \\
\hline All size of PTC $(n)$ & 1141 & 581 & 1722 & \\
\hline Age at diagnosis & $45.25 \pm 13.63$ & $41.40 \pm 13.26$ & & $<0.01$ \\
\hline$<45 \mathrm{y}$ & $560(49.1 \%)$ & $359(61.8 \%)$ & $919(53.4 \%)$ & $<0.01$ \\
\hline$\geqslant 45 y$ & $581(50.9 \%)$ & $222(38.2 \%)$ & $803(46.6 \%)$ & \\
\hline \multicolumn{5}{|l|}{ Gender } \\
\hline Male & $301(26.4 \%)$ & $46(7.9 \%)$ & $347(20.2 \%)$ & $<0.01$ \\
\hline Female & $840(73.6 \%)$ & $535(92.1 \%)$ & $1375(79.8 \%)$ & \\
\hline Number of positive lymph nodes & $3.05 \pm 0.192$ & $2.66 \pm 0.235$ & & 0.21 \\
\hline Foci of tumor & $1.46 \pm 0.025$ & $1.46 \pm 0.032$ & & 0.91 \\
\hline Maximum diameter $(\mathrm{mm})$ & $15.08 \pm 0.358$ & $13.92 \pm 0.421$ & & 0.05 \\
\hline Bilateral tumor & $241(21.2 \%)$ & $140(24.1 \%)$ & 381 & 0.16 \\
\hline Positive LNM & $366(32.1 \%)$ & $179(30.8 \%)$ & 545 & 0.60 \\
\hline $\operatorname{mPTC}(n)$ & 541 & 282 & 822 & \\
\hline Age at diagnosis & $47.21 \pm 12.03$ & $43.09 \pm 13.04$ & & $<0.01$ \\
\hline$<45 y$ & $226(41.8 \%)$ & $159(56.4 \%)$ & 385 & $<0.01$ \\
\hline$\geqslant 45 y$ & $315(58.2 \%)$ & $123(43.6)$ & 438 & \\
\hline \multicolumn{5}{|l|}{ Gender } \\
\hline Male & $121(22.4 \%)$ & $20(7.1 \%)$ & 141 & $<0.01$ \\
\hline Female & $420(77.6 \%)$ & $262(92.9 \%)$ & 682 & \\
\hline Number of positive lymph nodes & $1.71 \pm 0.196$ & $1.46 \pm 0.237$ & & 0.42 \\
\hline Foci of tumor & $1.32 \pm 0.030$ & $1.40 \pm 0.043$ & & 0.11 \\
\hline Maximum diameter (mm) & $6.02 \pm 0.122$ & $6.43 \pm 0.165$ & & 0.05 \\
\hline Bilateral tumor & $66(12.2 \%)$ & $52(18.4 \%)$ & 118 & 0.02 \\
\hline Positive LNM & $116(21.4 \%)$ & $57(20.2 \%)$ & 173 & 0.68 \\
\hline $\operatorname{crPTC}(n)$ & 600 & 299 & 899 & \\
\hline Age at diagnosis & $43.48 \pm 14.72$ & $39.80 \pm 13.29$ & & $<0.01$ \\
\hline$<45 y$ & $334(55.7 \%)$ & $200(66.9 \%)$ & 534 & $<0.01$ \\
\hline$\geqslant 45 y$ & $266(44.3 \%)$ & $99(33.1 \%)$ & 365 & \\
\hline \multicolumn{5}{|l|}{ Gender } \\
\hline Male & $180(30.0 \%)$ & $26(8.7 \%)$ & 206 & \\
\hline Female & $420(70.0 \%)$ & $273(91.3 \%)$ & 693 & $<0.01$ \\
\hline Number of positive lymph nodes & $3.97 \pm 0.285$ & $3.62 \pm 0.363$ & & 0.46 \\
\hline Foci of tumor & $1.59 \pm 0.039$ & $1.51 \pm 0.046$ & & 0.20 \\
\hline Maximum diameter (mm) & $23.26 \pm 0.467$ & $20.98 \pm 0.549$ & & $<0.01$ \\
\hline Bilateral tumor & $175(29.3 \%)$ & $88(29.5 \%)$ & 263 & 0.93 \\
\hline Positive LNM & $250(41.7 \%)$ & $122(40.8 \%)$ & 372 & 0.80 \\
\hline
\end{tabular}

Data are expressed as number (percentage) or mean $\pm \mathrm{SD}$. 
In this regard, we evaluated 6,432 consecutive thyroidectomies over 6 years from a single referral institution in China, revealing a unique association between Hashimoto's thyroiditis and PTC. Particularly, Hashimoto's thyroiditis increases the risk of papillary thyroid carcinoma in affected subjects, though it appears to impart no risk factor upon lymph node metastasis and progressive disease. This association was true both for mPTC and for crPTC, though it was not detected for all other thyroid malignancies including follicular, medullary, and anaplastic thyroid carcinoma. These data support previous pilot findings and suggest those with HT may warrant increased attention for the development of clinically relevant thyroid nodules given their higher risk of malignancy.

A recent meta-analysis of this topic similarly demonstrated that Hashimoto's thyroiditis is correlated to the presence of PTC, with carcinoma detected in 9.46 36.60\% of patients with HT. In our study, the prevalence of PTC in the HT cohort was $43.8 \%$, while in the non-HT group the prevalence was $22.4 \%$. Zhang showed in areas where the prevalence of HT was even higher the prevalence of PTC in HT was also higher, reaching $58.3 \%$ compared to $44.3 \%$ in non-HT group in the same region [22]. This suggests a similar influence of Hashimoto's disease upon cancer risk across different populations despite variable incidence of malignancy. Our study showed that HT was not only closely related to an increased risk of crPTC, but also significantly related to the risk of $\mathrm{mPTC}$. This similar relationship to both crPTC and mPTC strengthens the translatability of our data and lessens concern for selection bias in our study population. Beyond its clinical importance, our data also lends support to the hypothesis that microcellular dynamics (such as inflammation) impact the development of thyroid malignancy independent of nodule size.

Interestingly, although the coexistence of Hashimoto's disease appears related to increased risk of PTC, our results showed HT was not a risk factor for LNM in PTCs. And the maximum diameter of PTC seemed to be related to HT in mPTC stage and conversely in the crPTC stage. The mechanism behind this observation remains uncertain. In support, however, Dvorkin has previously found that differentiated thyroid cancer is similarly associated with less aggressive disease and better outcome in patients with coexisting HT, while Zhou has also found that HT is not a risk factor for occult contralateral carcinoma in patients with unilateral mPTCs [23]. Increasingly, clinical and basic investigations appear to confirm the important influence of the body's immune system in relation to malignant disease. While infection and immune response have been associated with an increased risk of malignancy in illnesses such as hepatitis, it is equally clear that immunologic influence can also positively influence malignant progression. This is perhaps best exemplified by melanoma or breast carcinoma, where numerous case reports have described unusual, prolonged remissions or late recurrences which implicate the immune system as a causative factor. Thus, while speculative, it is not unreasonable to postulate that chronic inflammation of the thyroid may increase malignant transformation, while nonetheless stimulating an immunologic milieu in which tumor expansion and malignant growth are less favored. Clinically, such a hypothesis would be nearly impossible to prove, as there currently exist no immunomodulating therapies for Hashimoto's disease by which one could study cancer prevalence and progression following a randomized intervention. However, it is also possible that HT may be the "response" to the tumor in genetically predisposed subjects and that this response may slow down cancer progression. Regardless, these data nonetheless provide insight into the complex interactions of thyroid cellular biochemistry and immunology and provide a stimulus for further investigation. We did find that male gender, age $<45$ years, foci of tumor, and maximum diameter were risk factors for LNM in PTCs in accordance with another previous study [24].

The strength of our study relates to the size and uniformity of our study cohort, as well as the strict histopathologic definitions used for both Hashimoto's thyroiditis and thyroid malignancy. We also analyzed PTC across the full spectrum of size, including evaluation of microcarcinomas no more than $1 \mathrm{~cm}$ in diameter. The similar relationship of HT to both mPTC and crPTC is notable and speaks against selection or sampling bias. Nonetheless, we acknowledge the limitations to our investigation. Our study was retrospective and from a single study center. We tried to eliminate further selection bias by subanalyzing our total cohort by gender, age, size of tumor, and using binary logistic regression method. We also note that a relatively large percent $(69.3 \%)$ of benign nodules was resected. Most often, this was simply due to nodule size more than $3 \mathrm{~cm}$ or the presence of large multinodular goiters (data not shown). While an ideal study would prospectively investigate thyroidectomy histopathology among all patients with nodular disease, it is unreasonable to routinely perform thyroidectomies on all low risk patients [21], or those with benign disease. We also note that HT itself was not a thyroidectomy indication in our study. Recent data confirm modest interobserver variability in cancer diagnosis [25], which could reasonably suggest slight differences in translation of these data at other institutions.

In conclusion, these data provide the most extensive consecutive analysis of the relationship between Hashimoto's thyroiditis and thyroid carcinoma, confirming an increased risk of PTC in the setting of HT. While this increased risk appears independent of PTC size, however, during more advanced stage, HT was no longer a risk factor suggesting a potential protective effect of the inflammatory milieu upon the progression of malignancy as defined by local LNM. These data support previous pilot findings and suggest that patients with HT may warrant increased attention for the development of clinically relevant thyroid nodules given their higher risk of malignancy.

\section{Conflict of Interests}

All authors report no conflict of interests relevant to this work. Dr. Alexander has served as a consultant to Genzyme, Inc., and Veracyte, Inc., and is on the Scientific Advisory Board of Asuragen, Inc. 


\section{Authors' Contribution}

Xiaoyun Liu and Lijun Zhu contributed equally to this work.

\section{Acknowledgments}

The authors thank Professor Edmund S. Cibas, M.D., from Department of Pathology, Brigham \& Women's Hospital and Harvard Medical School, for review of the paper and helpful comments. This work has been presented as a poster presentation at ICE/ENDO 2014, June 21-24 in Chicago, Illinois. This work was supported by National Science Foundation of China (nos. 81270897, 30971405, and 30671010) and Priority Academic Program Development of Jiangsu Higher Education Institutions (PAPD) as well as Jiangsu Health International Exchange Program (Xiaoyun Liu).

\section{References}

[1] J. R. Burgess, "Temporal trends for thyroid carcinoma in Australia: an increasing incidence of papillary thyroid carcinoma (1982-1997)," Thyroid, vol. 12, no. 2, pp. 141-149, 2002.

[2] L. Davies and H. G. Welch, "Increasing incidence of thyroid cancer in the United States, 1973-2002," The Journal of the American Medical Association, vol. 295, no. 18, pp. 2164-2167, 2006.

[3] L. Leenhardt, P. Grosclaude, and L. Chérié-Challine, "Increased incidence of thyroid carcinoma in france: a true epidemic or thyroid nodule management effects? Report from the french thyroid cancer committee," Thyroid, vol. 14, no. 12, pp. 10561060, 2004.

[4] Y. Ito, Y. E. Nikiforov, M. Schlumberger, and R. Vigneri, "Increasing incidence of thyroid cancer: controversies explored," Nature Reviews Endocrinology, vol. 9, no. 3, pp. 178-184, 2013.

[5] D. T. Hughes, M. R. Haymart, B. S. Miller, P. G. Gauger, and G. M. Doherty, "The most commonly occurring papillary thyroid cancer in the United States is now a microcarcinoma in a patient older than 45 years," Thyroid, vol. 21, no. 3, pp. 231-236, 2011.

[6] Y. Wang and W. Wang, "Increasing incidence of thyroid cancer in Shanghai, China, 1983-2007," Asia-Pacific Journal of Public Health, 2012.

[7] K.-W. Jung, S. Park, H.-J. Kong et al., "Cancer statistics in Korea: incidence, mortality, survival, and prevalence in 2009," Cancer Research and Treatment, vol. 44, no. 1, pp. 11-24, 2012.

[8] J. Xiang, Y. Wu, D. S. Li et al., "New clinical features of thyroid cancer in Eastern China," Journal of Visceral Surgery, vol. 147, no. 1, pp. e53-e56, 2010.

[9] X. Y. Liu, L. J. Zhu, D. Cui et al., "Annual financial impact of thyroidectomies for nodular thyroid disease in China," Asian Pacific Journal of Cancer Prevention, vol. 15, no. 14, pp. 59215926, 2014.

[10] R. Siegel, D. Naishadham, and A. Jemal, "Cancer statistics, 2012," CA Cancer Journal for Clinicians, vol. 62, no. 1, pp. 10-29, 2012.

[11] D. Ahn, S. J. Heo, J. H. Park et al., "Clinical relationship between Hashimoto's thyroiditis and papillary thyroid cancer," Acta Oncologica, vol. 50, no. 8, pp. 1228-1234, 2011.

[12] E. Fiore, T. Rago, F. Latrofa et al., "Hashimoto's thyroiditis is associated with papillary thyroid carcinoma: role of TSH and of treatment with L-thyroxine," Endocrine-Related Cancer, vol. 18, no. 4, pp. 429-437, 2011.
[13] D. Repplinger, A. Bargren, Y.-W. Zhang, J. T. Adler, M. Haymart, and H. Chen, "Is Hashimoto's thyroiditis a risk factor for papillary thyroid cancer?" Journal of Surgical Research, vol. 150, no. 1, pp. 49-52, 2008.

[14] C. Cipolla, L. Sandonato, G. Graceffa et al., "Hashimoto thyroiditis coexistent with papillary thyroid carcinoma," The American Surgeon, vol. 71, no. 10, pp. 874-878, 2005.

[15] B. Jankovic, K. T. Le, and J. M. Hershman, "Hashimoto's thyroiditis and papillary thyroid carcinoma: is there a correlation?" Journal of Clinical Endocrinology and Metabolism, vol. 98, no. 2, pp. 474-482, 2013.

[16] Y. Lun, X. Wu, Q. Xia et al., "Hashimoto's thyroiditis as a risk factor of papillary thyroid cancer may improve cancer prognosis," Otolaryngology-Head and Neck Surgery, vol. 148, no. 3, pp. 396-402, 2013.

[17] K. W. Kim, Y. J. Park, E. H. Kim et al., "Elevated risk of papillary thyroid cancer in Korean patients with Hashimoto's thyroiditis," Head and Neck, vol. 33, no. 5, pp. 691-695, 2011.

[18] S. Dvorkin, E. Robenshtok, D. Hirsch, Y. Strenov, I. Shimon, and C. A. Benbassat, "Differentiated thyroid cancer is associated with less aggressive disease and better outcome in patients with coexisting hashimotos thyroiditis," The Journal of Clinical Endocrinology and Metabolism, vol. 98, no. 6, pp. 2409-2414, 2013.

[19] C. Hedinger, E. D. Williams, and L. H. Sobin, "The WHO histological classification of thyroid tumors: a commentary on the second edition," Cancer, vol. 63, no. 5, pp. 908-911, 1989.

[20] J. A. Sosa and R. Udelsman, "Papillary thyroid cancer," Surgical Oncology Clinics of North America, vol. 15, no. 3, pp. 585-601, 2006.

[21] American Thyroid Association (ATA) Guidelines Taskforce on Thyroid Nodules and Differentiated Thyroid Cancer, D. S. Cooper, G. M. Doherty et al., "Revised American Thyroid Association management guidelines for patients with thyroid nodules and differentiated thyroid cancer," Thyroid, vol. 19, no. 11, pp. 1167-1214, 2009.

[22] L. Zhang, H. Li, Q.-H. Ji et al., “The clinical features of papillary thyroid cancer in Hashimoto's thyroiditis patients from an area with a high prevalence of Hashimoto's disease," BMC Cancer, vol. 12, article 610, 2012.

[23] Y.-L. Zhou, W. Zhang, E.-L. Gao et al., "Preoperative BRAF mutation is predictive of occult contralateral carcinoma in patients with unilateral papillary thyroid microcarcinoma," Asian Pacific Journal of Cancer Prevention, vol. 13, no. 4, pp. 1267-1272, 2012.

[24] L. Zhang, W.-J. Wei, Q.-H. Ji et al., "Risk factors for neck nodal metastasis in papillary thyroid microcarcinoma: a study of 1066 patients," Journal of Clinical Endocrinology and Metabolism, vol. 97, no. 4, pp. 1250-1257, 2012.

[25] E. S. Cibas, Z. W. Baloch, G. Fellegara et al., "A prospective assessment defining the limitations of thyroid nodule pathologic evaluation," Annals of Internal Medicine, vol. 159, no. 5, pp. 325-332, 2013. 


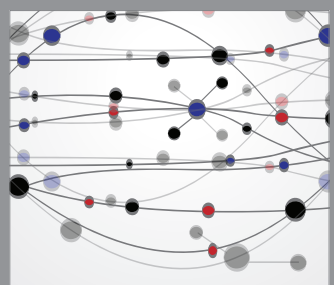

The Scientific World Journal
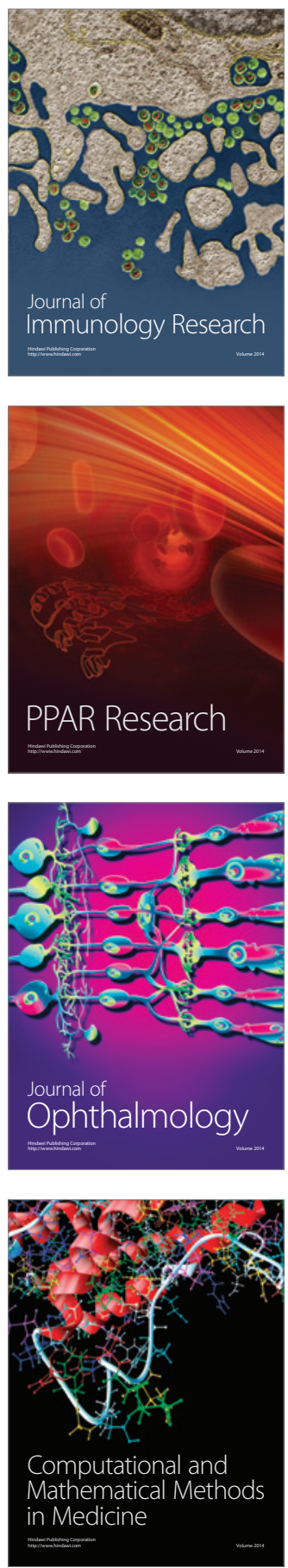

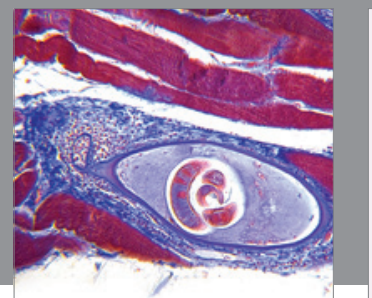

Gastroenterology

Research and Practice
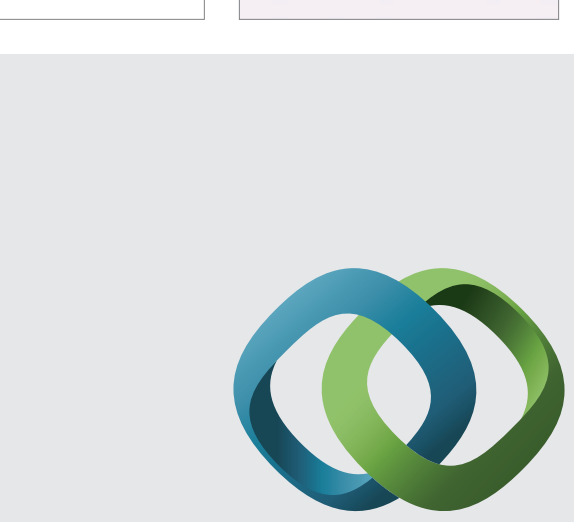

\section{Hindawi}

Submit your manuscripts at

http://www.hindawi.com
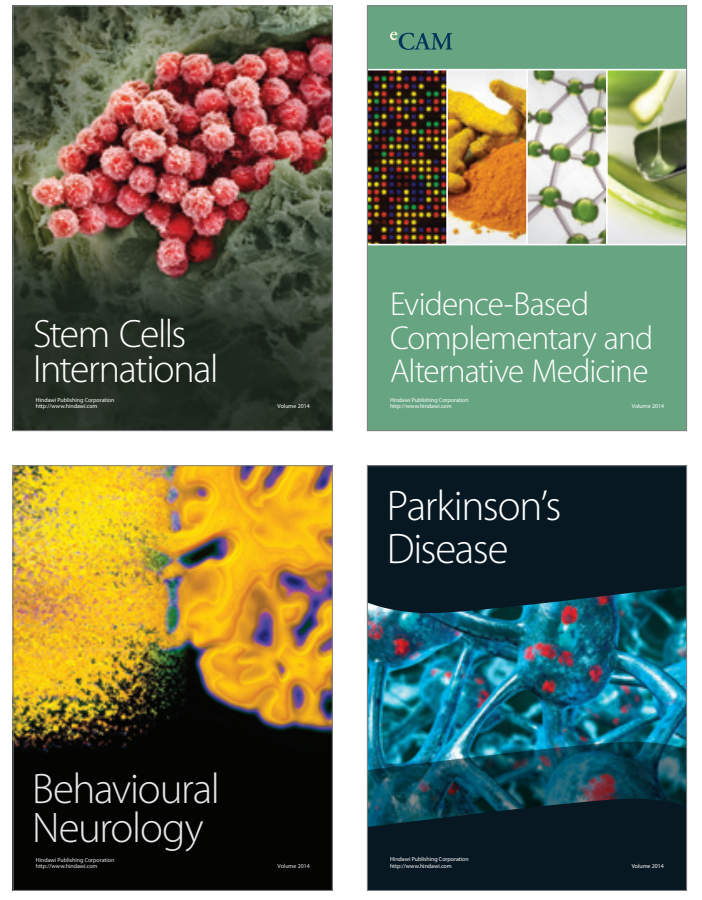
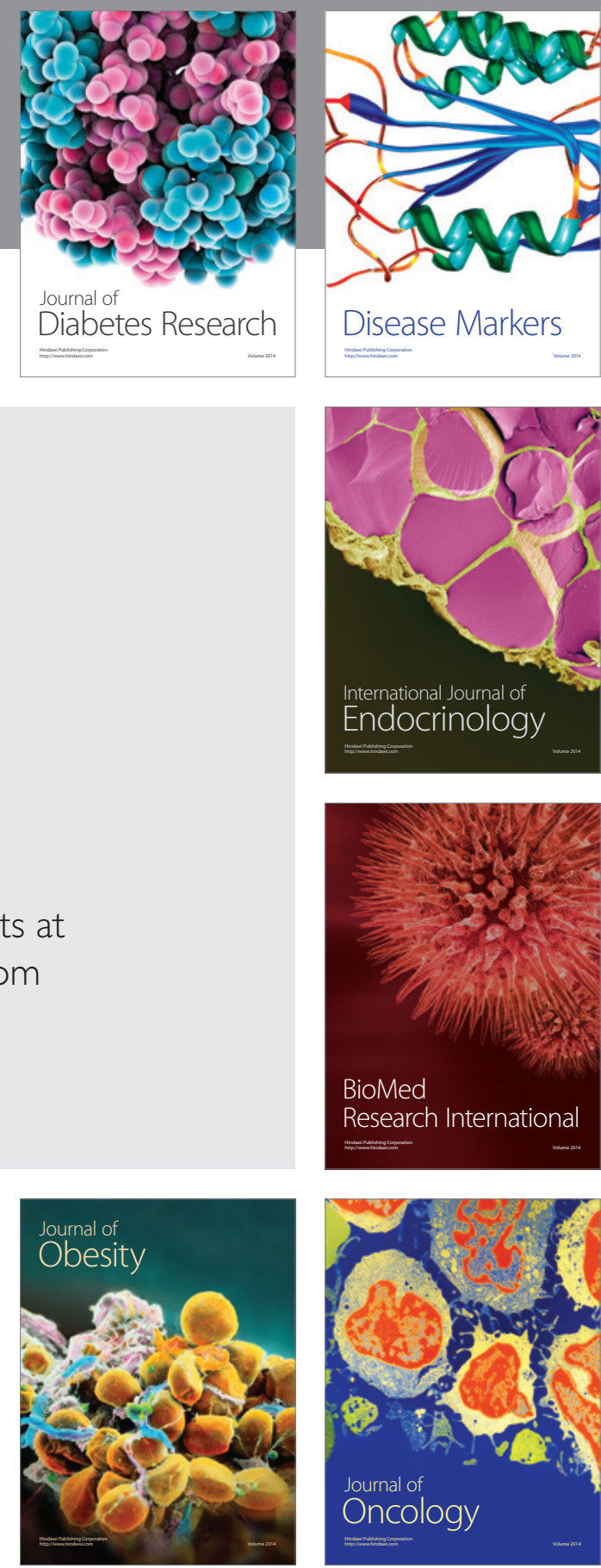

Disease Markers
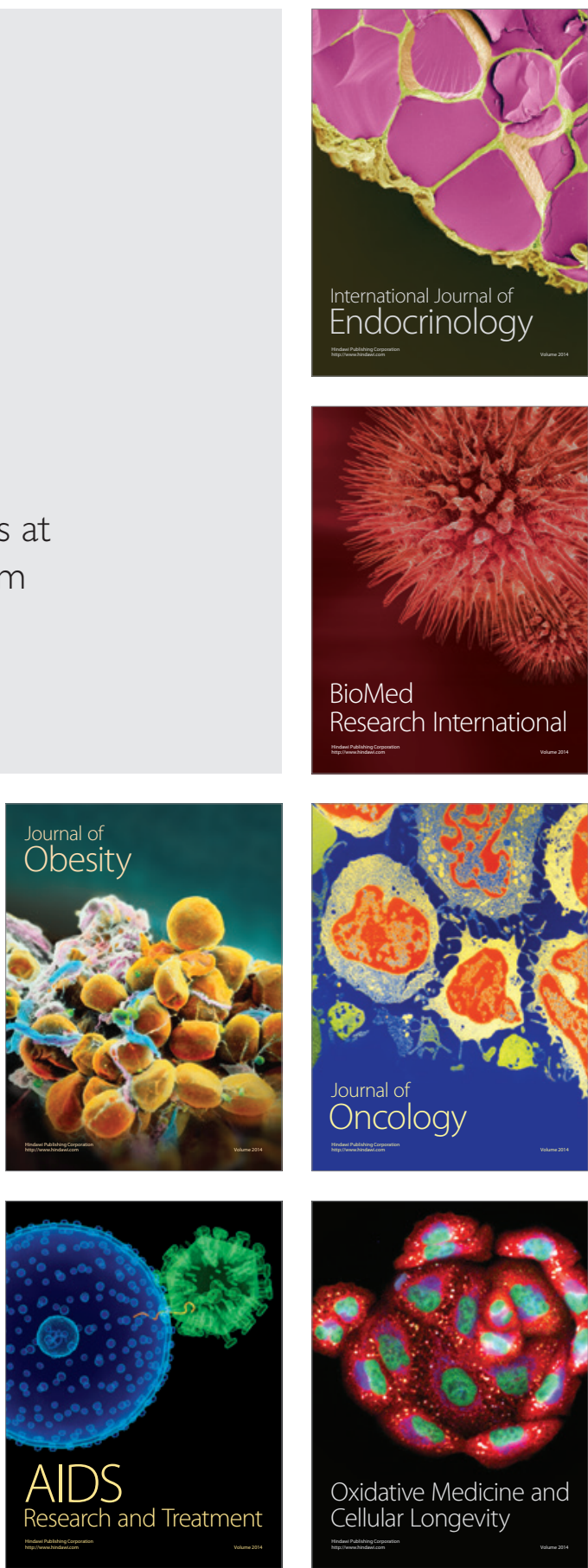